\title{
Effects of two-phase transport in the cathode gas diffusion layer on the performance of a PEMFC
}

\author{
Min-Hsing Chang ${ }^{\mathrm{a}, *}$, Falin Chen ${ }^{\mathrm{b}}$, Hong-She Teng ${ }^{\mathrm{b}}$ \\ ${ }^{a}$ Department of Mechanical Engineering, Yung-Ta Institute of Technology and Commerce, PingTung 909, Taiwan, ROC \\ ${ }^{\mathrm{b}}$ Institute of Applied Mechanics, National Taiwan University, Taipei 10764, Taiwan, ROC \\ Received 9 December 2005; received in revised form 27 December 2005; accepted 6 January 2006 \\ Available online 17 February 2006
}

\begin{abstract}
This study investigates the characteristics of transport phenomena in the cathode gas diffusion layer (GDL) of a proton exchange membrane fuel cell (PEMFC) and their influences on cell performance by utilizing a two-phase flow model based on a multiphase mixture formulation. The profiles of liquid water saturation and oxygen concentration across the GDL are obtained by a direct numerical procedure, and the corresponding polarization curves are determined as well. The results show that the liquid water generated by the electrochemical reaction could significantly reduce the effective porosity of the GDL under high current density conditions to hinder oxygen transport through the GDL. The removal rate of liquid water due to capillarity-induced motion can be enhanced conspicuously by an increase in hydrophobicity at first, and this effect then diminishes gradually. The effects of porosity and thickness of the GDL, inlet velocity of reactant gas, and gas channel length on the cell performance are also examined in detail. The results provide insights into the transport mechanisms in the GDL and benefit the design of PEMFCs.
\end{abstract}

(c) 2006 Elsevier B.V. All rights reserved.

Keywords: PEM fuel cell; Cathode gas diffusion layer; Two-phase flow

\section{Introduction}

The proton exchange membrane fuel cell (PEMFC) is considered as an alternative power source for widely promising applications in many power systems. However, due to its lower operating temperature and the utilization of external humidification of the reactant gases, water management is quite important to avoid the so-called "flooding" phenomenon, particularly on the cathode side where the water vapor may condense and block the pores of the GDL, and so reduce the amount of oxygen transport to the catalyst layer. Such a limiting factor in PEMFC performance is well-known, and thus it is vital to understand the two-phase transport characteristics within the cathode GDL and their influences on the cell performance.

Numerous studies have devoted to developing mathematical models for the transport of reactants and product water in a PEMFC [1-11]. Particularly, since it involves a complicated two-phase transport process in the GDL, several two-phase flow

\footnotetext{
* Corresponding author. Tel.: +886 8 7233733; fax: +886 87215649 .

E-mail address: mhchang@mail.ytit.edu.tw (M.-H. Chang).
}

models for the cathode of PEMFC have been proposed to simulate the effects of the gas and liquid water hydrodynamics on cell performance. He et al. [12] developed a two-phase flow model for PEMFCs with interdigitated flow fields. In this model, they considered the transport of liquid water through the completely wetted GDL which is driven by the shear force of gas flow and capillary force. Berning and Djilali [13] presented a 3D, multiphase, and multicomponent model for the anode and cathode of a PEMFC. They described the two-phase flow inside the GDL by the unsaturated flow theory (UFT) that a uniform gas-phase pressure is assumed within the GDL. In contrast to the traditional UFT, Wang and Cheng [14,15] proposed a multiphase mixture $\left(\mathrm{M}^{2}\right)$ model that the assumption of constant gas pressure is released and it accounts for the gas flow counter to the capillarity-driven liquid flow. This $\mathrm{M}^{2}$ model has been widely used recently to investigate the multiphase and multicomponent transport in cathode GDL [16-21]. Wang et al. [16] performed a study for the two-phase transport of reactants and products in the cathode of a PEMFC. They considered single and two-phase regimes of water distribution and classified them by a threshold current density corresponding to the emergence of liquid water at the membrane/cathode interface. 
Natarajan and Nguyen [17] developed a 2D two-phase transient model for the cathode of a PEMFC using conventional gas distributors and found that the liquid water transport is the slowest mass transfer phenomenon in the cathode which is mainly responsible for mass transfer restrictions. A two-phase mixture model was also reported by You and Liu [18], and accordingly they obtained some typical two-phase flow distributions in the cathode GDL and gas channel. They also discussed the main parameters influencing water transport across the membrane. Pasaogullari and Wang [19] studied the liquid water transport in both hydrophilic and hydrophobic GDL of PEMFCs. They made some simplifications and then derived a one-dimensional analytical solution for liquid water transport across the GDL. A detailed exploration for the effects of GDL wettability on liquid water transport was also given in this work. Moreover, they used the $\mathrm{M}^{2}$ model to investigate the effects of counter gas flow with respect to the liquid water flow [20] and found that it should be a new oxygen transport mechanism. They further considered the two-phase transport in PEMFCs with multilayer diffusion media [20,21] and examined the properties of the micro-porous layer including average pore size, wettability, thickness, and porosity.

In the present study, a two-phase flow model based on the multiphase mixture concept is developed to investigate the transport characteristics in a hydrophobic GDL of PEMFC. In addition to the variations of liquid water saturation and oxygen concentration profiles across the GDL, the effects of gas inlet velocity, gas channel length, and GDL properties including wettability, thickness, and porosity on the polarization curve of a PEMFC were also examined in detail. The results give an overview for the influences of two-phase transport in GDL on the cell performance.

\section{Mathematical model}

\subsection{Governing equations}

The modeling domain for the cathode GDL is shown in Fig. 1. The humidified air enters the gas channel at a uni- form velocity $u_{\text {in }}$, where $L$ is the gas channel length, $H_{1}$ the gas channel thickness $\left(H_{2}-H_{1}\right)$ and $\left(H_{3}-H_{2}\right)$ are the thicknesses of gas diffusion layer and catalyst layer, respectively. To investigate the two-phase transport within the GDL, the following assumptions are made to simplify the model development:

1. The system is isothermal and under steady state.

2. The gas phase is an ideal gas mixture and insoluble in the liquid phase.

3. The catalyst layer is infinitely thin and can be treated as a boundary where electrochemical reaction occurs.

4. The membrane is fully hydrated by liquid water.

According to the multiphase mixture $\left(\mathrm{M}^{2}\right)$ model and above assumptions, the continuity equation for the mixture in the GDL can be written as

$\varepsilon \frac{\partial \rho}{\partial t}+\nabla \cdot(\rho \mathbf{u})=0$

where $\varepsilon$ is the porosity, $\rho$ the two-phase mixture density, and $\mathbf{u}$ the superficial two-phase mixture velocity. The species conservation equation for this two-phase flow problem [14] is given as

$$
\begin{aligned}
& \varepsilon \frac{\partial}{\partial t}\left(\rho C^{\alpha}\right)+\nabla \cdot\left(\gamma_{\alpha} \rho \mathbf{u} C^{\alpha}\right) \\
& =\nabla \cdot\left(\varepsilon \rho D^{\alpha} \nabla C^{\alpha}\right)+\nabla \cdot\left[\sum_{k} \varepsilon \rho_{k} s_{k} D_{k}^{\alpha}\left(\nabla C_{k}^{\alpha}-\nabla C^{\alpha}\right)\right] \\
& \quad-\nabla \cdot\left(\sum_{k} C_{k}^{\alpha} \mathbf{j}_{k}\right),
\end{aligned}
$$

where $C$ is the species mass concentration, $D$ the diffusion coefficient, $s$ the phase saturation, and the superscript $\alpha$ and subscript $k$ denote the species and phase, respectively. The vector $\mathbf{j}$ is the diffusive mass flux and $\gamma$ is the advection correction factor

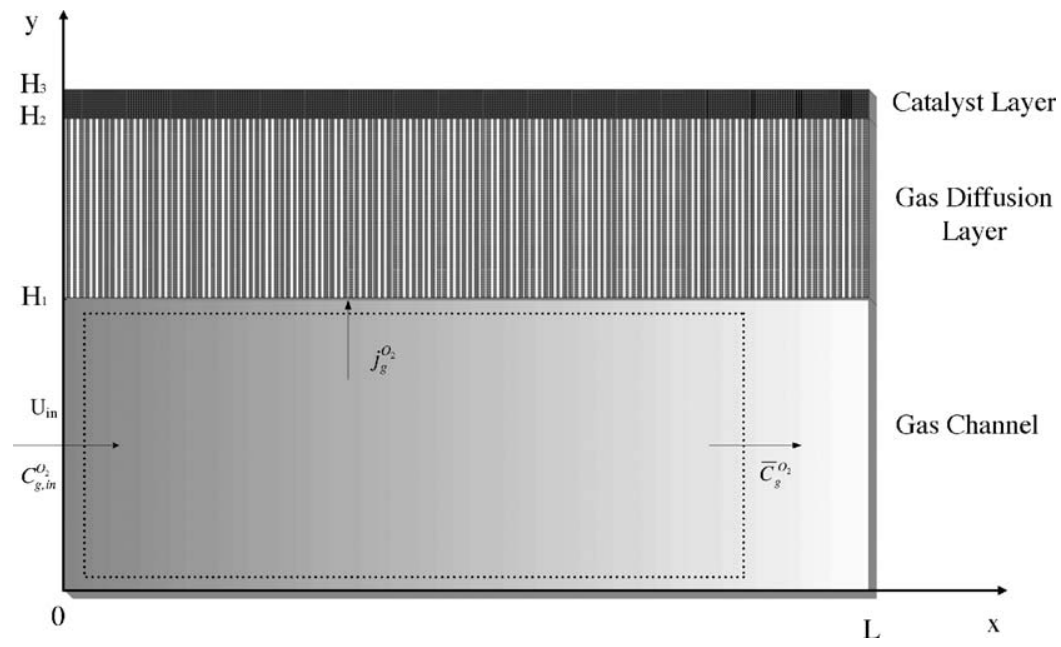

Fig. 1. Schematic diagram of the model for the cathode side of PEMFC. 
defined by

$\gamma_{\alpha}=\frac{\rho \sum_{k} \lambda_{k} C_{k}^{\alpha}}{\sum_{k} \rho_{k} s_{k} C_{k}^{\alpha}}$,

where $\lambda_{k}$ is the mobility of phase $k$. Since this study is dealing with a two-phase flow problem, the parameter $k$ is equal to 2 including both liquid and gas phases. Consequently, Eq. (2) can be reduced to

$$
\begin{aligned}
\varepsilon & \frac{\partial}{\partial t}\left(\rho C^{\alpha}\right)+\nabla \cdot\left(\gamma_{\alpha} \rho \mathbf{u} C^{\alpha}\right) \\
= & \nabla \cdot\left(\varepsilon \rho D^{\alpha} \nabla C^{\alpha}\right)+\varepsilon \nabla \cdot\left[\rho_{1} s D_{1}^{\alpha} \nabla C_{1}^{\alpha}+\rho_{\mathrm{g}}(1-s) D_{\mathrm{g}}^{\alpha} \nabla C_{\mathrm{g}}^{\alpha}\right. \\
& \left.\quad-\rho D^{\alpha} \nabla C^{\alpha}\right]-\nabla \cdot\left[\left(C_{1}^{\alpha}-C_{\mathrm{g}}^{\alpha}\right) \mathbf{j}_{1}\right] .
\end{aligned}
$$

Note that here $s$ denotes the liquid water saturation that represents the volume ratio of the liquid water to the void space of the GDL. The quantities of the mixture in the above equation are defined as

$\rho=\rho_{\mathrm{l}} s+\rho_{\mathrm{g}}(1-s)$,

$\rho C^{\alpha}=\rho_{\mathrm{l}} s C_{1}^{\alpha}+\rho_{\mathrm{g}}(1-s) C_{\mathrm{g}}^{\alpha}$,

$\rho \mathbf{u}=\rho_{\mathrm{l}} \mathbf{u}_{1}+\rho_{\mathrm{g}} \mathbf{u}_{\mathrm{g}}$,

$\rho D^{\alpha}=\rho_{\mathrm{l}} s D_{1}^{\alpha}+\rho_{\mathrm{g}}(1-s) D_{\mathrm{g}}^{\alpha}$,

and $\gamma_{\alpha}$ becomes:

$\gamma_{\alpha}=\frac{\rho\left(\lambda_{1} C_{1}^{\alpha}+\lambda_{\mathrm{g}} C_{\mathrm{g}}^{\alpha}\right)}{\rho_{\mathrm{l}} s C_{1}^{\alpha}+\rho_{\mathrm{g}}(1-s) C_{\mathrm{g}}^{\alpha}}$,

where the mobilities $\gamma_{1}$ and $\gamma_{\mathrm{g}}$ are defined as

$\lambda_{1}=\frac{k_{\mathrm{rl}} / \nu_{\mathrm{l}}}{k_{\mathrm{rl}} / \nu_{\mathrm{l}}+k_{\mathrm{rg}} / \nu_{\mathrm{g}}}$,

$\lambda_{\mathrm{g}}=\frac{k_{\mathrm{rg}} / \nu_{\mathrm{g}}}{k_{\mathrm{rl}} / v_{\mathrm{l}}+k_{\mathrm{rg}} / v_{\mathrm{g}}}$.

The parameters $k_{\mathrm{rl}}$ and $k_{\mathrm{rg}}$ are the relative permeability of the liquid and gas phase given by empirical correlation [22]:

$k_{\mathrm{rl}}=s^{3}$,

$k_{\mathrm{rg}}=(1-s)^{3}$.

The diffusive mass flux of liquid phase $\mathbf{j}_{1}$ can be given as [15]:

$\mathbf{j}_{1}=\frac{K \lambda_{1} \lambda_{\mathrm{g}}}{v}\left[\nabla P_{\mathrm{c}}+\left(\rho_{\mathrm{l}}-\rho_{\mathrm{g}}\right) \mathbf{g}\right]$,

where $K$ is the absolute permeability, $P_{\mathrm{c}}$ the capillary pressure, and $v$ the mixture kinematic viscosity defined by

$v=\frac{1}{k_{\mathrm{rl}} / v_{1}+k_{\mathrm{rg}} / \nu_{\mathrm{g}}}$.

In general, the gravity term in Eq. (14) is negligible in comparison with the capillary effect, so $\mathbf{j}_{1}$ can be expressed by

$\mathbf{j}_{1}=\frac{K \lambda_{1} \lambda_{\mathrm{g}}}{v} \nabla P_{\mathrm{c}}$ and the capillary pressure between the gas and liquid phases can be related to the liquid phase saturation [15] as

$P_{\mathrm{c}}=\sigma \cos \left(\theta_{\mathrm{c}}\right)\left(\frac{\varepsilon}{K}\right)^{1 / 2} J(s)$,

where $\sigma$ is the surface tension of liquid water, $\theta_{\mathrm{c}}$ the contact angle, and $J(s)$ is the Leverett function [19]. For hydrophilic $\operatorname{GDL}\left(0^{\circ}<\theta_{\mathrm{c}}<90^{\circ}\right), J(s)$ is given by

$J(s)=1.417(1-s)-2.12(1-s)^{2}+1.263(1-s)^{3}$,

while for hydrophobic GDL $\left(90^{\circ}<\theta_{\mathrm{c}}<180^{\circ}\right), J(s)$ is

$J(s)=1.417 s-2.12 s^{2}+1.263 s^{3}$.

Substituting Eq. (17) in Eq. (16), we can obtain:

$\mathbf{j}_{1}=\frac{K \lambda_{1} \lambda_{\mathrm{g}}}{v} \sigma \cos \left(\theta_{\mathrm{c}}\right)\left(\frac{\varepsilon}{K}\right)^{1 / 2} \frac{\mathrm{d} J(s)}{\mathrm{d} s} \nabla s$.

\subsection{Water transport}

When the vapor partial pressure exceeds the corresponding saturation vapor pressure, the vapor condenses and the concentration of water vapor in the gas mixture is

$C_{\mathrm{g}}^{\mathrm{H}_{2} \mathrm{O}}=\frac{\rho_{\mathrm{v}}^{\mathrm{H}_{2} \mathrm{O}}}{\rho_{\mathrm{g}}}=\frac{P_{\mathrm{v}} M^{\mathrm{H}_{2} \mathrm{O}}}{P M^{\text {air }}}$.

Assuming that oxygen and nitrogen are insoluble in the liquid phase, the concentration of liquid water is

$C_{1}^{\mathrm{H}_{2} \mathrm{O}}=1$.

Since the GDL is isothermal and under steady state, if the GDL is assumed to be saturated with water vapor, the concentration of water vapor is uniform and the gas phase diffusion vanishes. Accordingly, substituting Eqs. (21) and (22) into Eq. (4) yields:

$\nabla \cdot\left(\gamma_{\mathrm{H}_{2} \mathrm{O}} \rho \mathbf{u} C^{\mathrm{H}_{2} \mathrm{O}}\right)+\nabla \cdot\left[\left(1-\frac{\rho_{\mathrm{v}}^{\mathrm{H}_{2} \mathrm{O}}}{\rho_{\mathrm{g}}}\right) \mathbf{j}_{1}\right]=0$,

where the advection correction factor $\gamma_{\mathrm{H}_{2} \mathrm{O}}$ becomes:

$\gamma_{\mathrm{H}_{2} \mathrm{O}}=\frac{\lambda_{1} C_{1}^{\mathrm{H}_{2} \mathrm{O}}+\lambda_{\mathrm{g}} C_{\mathrm{g}}^{\mathrm{H}_{2} \mathrm{O}}}{C^{\mathrm{H}_{2} \mathrm{O}}}$.

Integrating the continuity equation (1) for the two-phase mixture over the GDL [20], we can obtain:

$\rho v=-\frac{I}{2 F}(1+2 \alpha) M^{\mathrm{H}_{2} \mathrm{O}}+\frac{I}{4 F} M^{\mathrm{O}_{2}}$,

where $I$ is the current density, $F$ the Faraday constant, $M$ the molecular weight, and $\alpha$ the net water transport coefficient across the membrane. A positive $\alpha$ means that the net water transport is from the anode side to the cathode side. Combining Eqs. (24) and (25) we have

$$
\begin{aligned}
\gamma_{\mathrm{H}_{2} \mathrm{O} \rho v C^{\mathrm{H}_{2} \mathrm{O}}=} & \left(\lambda_{1}+\lambda_{\mathrm{g}} \frac{\rho_{\mathrm{v}}^{\mathrm{H}_{2} \mathrm{O}}}{\rho_{\mathrm{g}}}\right) \\
& \times\left[-\frac{I}{2 F}(1+2 \alpha) M^{\mathrm{H}_{2} \mathrm{O}}+\frac{I}{4 F} M^{\mathrm{O}_{2}}\right] .
\end{aligned}
$$


Substituting Eqs. (20) and (26) into Eq. (23) and then integrating the resultant equation over the GDL, we can obtain the governing equation for the liquid water saturation:

$$
\begin{aligned}
\left(\lambda_{1}\right. & \left.+\lambda_{\mathrm{g}} \frac{\rho_{\mathrm{v}}^{\mathrm{H}_{2} \mathrm{O}}}{\rho_{\mathrm{g}}}\right)\left[-\frac{I}{2 F}(1+2 \alpha) M^{\mathrm{H}_{2} \mathrm{O}}+\frac{I}{4 F} M^{\mathrm{O}_{2}}\right] \\
& +\left(1-\frac{\rho_{\mathrm{v}}^{\mathrm{H}_{2} \mathrm{O}}}{\rho_{\mathrm{g}}}\right) \frac{K \lambda_{1} \lambda_{\mathrm{g}}}{v} \sigma \cos \left(\theta_{\mathrm{c}}\right)\left(\frac{\varepsilon}{K}\right)^{1 / 2} \frac{\mathrm{d} J(s)}{\mathrm{d} s} \nabla s \\
= & -\frac{I}{2 F} M^{\mathrm{H}_{2} \mathrm{O}}(1+2 \alpha) .
\end{aligned}
$$

\subsection{Oxygen transport}

It is assumed that oxygen is insoluble in the liquid phase, so we have

$C_{1}^{\mathrm{O}_{2}}=0$.

Consequently, Eq. (4) for oxygen becomes:

$\nabla \cdot\left(\gamma_{\mathrm{O}_{2}} \rho \mathbf{u} C^{\mathrm{O}_{2}}\right)=\nabla \cdot\left[\varepsilon \rho_{\mathrm{g}}(1-s) D_{\mathrm{g}}^{\mathrm{O}_{2}} \nabla C_{\mathrm{g}}^{\mathrm{O}_{2}}\right]+\nabla \cdot\left(C_{\mathrm{g}}^{\mathrm{O}_{2}} \mathbf{j}_{1}\right)$,

where the advection correction factor $\gamma_{\mathrm{O}_{2}}$ is

$\gamma_{\mathrm{O}_{2}}=\frac{\lambda_{\mathrm{g}} C_{\mathrm{g}}^{\mathrm{O}_{2}}}{C^{\mathrm{O}_{2}}}$

Combining Eq. (30) with Eq. (25), we have

$\gamma_{\mathrm{O}_{2}} \rho v C^{\mathrm{O}_{2}}=\lambda_{\mathrm{g}} C_{\mathrm{g}}^{\mathrm{O}_{2}}\left[-\frac{I}{2 F}(1+2 \alpha) M^{\mathrm{H}_{2} \mathrm{O}}+\frac{I}{4 F} M^{\mathrm{O}_{2}}\right]$.

Substituting Eqs. (20) and (31) into Eq. (29) and then integrating the resultant equation over the GDL thickness, one has

$$
\begin{gathered}
\lambda_{\mathrm{g}} C_{\mathrm{g}}^{\mathrm{O}_{2}}\left[-\frac{I}{2 F}(1+2 \alpha) M^{\mathrm{H}_{2} \mathrm{O}}+\frac{I}{4 F} M^{\mathrm{O}_{2}}\right] \\
-\left[C_{\mathrm{g}}^{\mathrm{O}_{2}} \frac{K \lambda_{1} \lambda_{\mathrm{g}}}{v} \sigma \cos \left(\theta_{\mathrm{c}}\right)\left(\frac{\varepsilon}{K}\right)^{1 / 2} \frac{\mathrm{d} J(s)}{\mathrm{d} s} \nabla s\right] \\
-\left[\varepsilon \rho_{\mathrm{g}}(1-s) D_{\mathrm{g}}^{\mathrm{O}_{2}, \text { eff }} \nabla C_{\mathrm{g}}^{\mathrm{O}_{2}}\right]=\frac{I}{4 F} M^{\mathrm{O}_{2}} .
\end{gathered}
$$

In above equation, the effective diffusion coefficient of oxygen $D_{\mathrm{g}}^{\mathrm{O}_{2} \text {,eff }}$ is modified by Bruggeman-type correlation [23] to consider the tortuosity factor:

$D_{\mathrm{g}}^{\mathrm{O}_{2}, \text { eff }}=[\varepsilon(1-s)]^{\tau} D_{\mathrm{g}}^{\mathrm{O}_{2}}$.

Eq. (32) is the governing equation of oxygen concentration. As shown, the transport of oxygen involves the advection, capillary force, and diffusion effects.

\subsection{Boundary conditions}

Both Eqs. (27) and (32) are first order nonlinear ordinary differential equation and two boundary conditions are needed to obtain the solution. Assuming the gas channel is free from liquid water and the liquid water saturation is zero at the interface between the gas channel and the GDL, we have

$s=0, \quad$ at $y=H_{1}$.

Furthermore, at this interface the mass flux of oxygen can be written in the form:

$j_{\mathrm{g}}^{\mathrm{O}_{2}}=-h_{\mathrm{m}} \rho_{\mathrm{g}}^{\mathrm{air}}\left(\left.C_{\mathrm{g}}^{\mathrm{O}_{2}}\right|_{y=H_{1}}-\bar{C}_{\mathrm{g}}^{\mathrm{O}_{2}}\right)=\frac{M^{\mathrm{O}_{2}} I}{4 F}$,

where $h_{\mathrm{m}}$ is the convective mass transfer coefficient and $\bar{C}_{\mathrm{g}}^{\mathrm{O}_{2}}$ is the local average concentration of oxygen. Note that the parameter $h_{\mathrm{m}}$ can be determined from the Sherwood number defined by [16]:

$S h=\frac{h_{\mathrm{m}} H_{1}}{D_{\mathrm{g}}^{\mathrm{O}_{2}}}=2.693$,

and the average oxygen concentration decreases along the channel due to electrochemical reaction. The mass flux of oxygen is assumed a constant along the channel. Accordingly, by using the approach of control volume integration within the dotted domain as shown in Fig. 1, we can obtain:

$u_{\text {in }} H_{1} \rho_{\mathrm{g}}^{\mathrm{air}}\left(\bar{C}_{\mathrm{g}}^{\mathrm{O}_{2}}-C_{\mathrm{g}, \mathrm{in}}^{\mathrm{O}_{2}}\right)=-\frac{M^{\mathrm{O}_{2}} I}{4 F} L_{1}$.

Combining Eqs. (35) and (37) yields the boundary condition for oxygen concentration at the interface $y=H_{1}$ :

$\left.C_{\mathrm{g}}^{\mathrm{O}_{2}}\right|_{y=H_{1}}=C_{\mathrm{g}, \text { in }}^{\mathrm{O}_{2}}-\frac{M^{\mathrm{O}_{2}} I}{4 \rho_{\mathrm{g}}^{\text {air } F}}\left(\frac{1}{h_{\mathrm{m}}}+\frac{L_{1}}{u_{\mathrm{in}} H_{1}}\right)$.

\subsection{Polarization curve}

The electrochemical reaction on the catalyst layer is described by the Tafel equation in the form:

$I=(1-s) I_{\mathrm{ref}} \frac{\left.C_{\mathrm{g}}^{\mathrm{O}_{2}}\right|_{y=H_{2}}}{C_{\mathrm{g}, \text { ref }}^{\mathrm{O}_{2}}} \exp \left(\frac{\alpha_{\mathrm{c}} F}{R T} \eta\right)$,

where $I_{\text {ref }}$ is the exchange current density, $\alpha_{\mathrm{c}}$ the cathode transfer coefficient, $F$ the Faraday constant, $R$ the universal gas constant, $T$ the absolute temperature, and $\eta$ is the overpotential. Here the term $(1-s)$ is used to account for the reduction of active surface due to liquid water coverage of catalyst particles. Hence, the output cell voltage can be determined by

$V=V_{\mathrm{oc}}-\eta-\eta_{\mathrm{ohm}}$

where $V_{\mathrm{oc}}$ is the open circuit voltage, and $\eta_{\mathrm{ohm}}$ is the ohmic losses. Assume that the ohmic losses are primary caused by the ion transport through the membrane and the membrane is fully saturated with liquid water, thus $\eta_{\mathrm{ohm}}$ can be approximated by

$\eta_{\mathrm{ohm}}=\frac{I H_{\mathrm{m}}}{\sigma_{\mathrm{m}}}$

where $H_{\mathrm{m}}$ and $\sigma_{\mathrm{m}}$ are the thickness and ion conductivity of the membrane, respectively.

Eqs. (27) and (32) together with the boundary conditions (34) and (38) can be solved by a fourth-order Runge-Kutta method 
Table 1

Physical properties and parameters

\begin{tabular}{|c|c|}
\hline Cell temperature, $T(\mathrm{~K})$ & 353 \\
\hline Air pressure, $P_{\mathrm{c}}(\mathrm{Pa})$ & $1.013 \times 10^{5}$ \\
\hline Universal gas constant, $R\left(\mathrm{~J} \mathrm{~mol}^{-1} \mathrm{~K}^{-1}\right)$ & 8.314 \\
\hline Faraday constant, $F$ & 96487 \\
\hline GDL porosity, $s$ & 0.5 \\
\hline Oxygen molecular weight, $M_{\mathrm{O}_{2}}\left(\mathrm{~kg} \mathrm{~mol}^{-1}\right)$ & 0.032 \\
\hline Water molecular weight, $M_{\mathrm{H}_{2} \mathrm{O}}\left(\mathrm{kg} \mathrm{mol}^{-1}\right)$ & 0.018 \\
\hline Oxygen diffusion coefficient, $D_{\mathrm{g}}^{\mathrm{O}_{2}}\left(\mathrm{~m}^{2} \mathrm{~s}^{-1}\right)$ & $1.805 \times 10^{-5}$ \\
\hline Oxygen mass fraction at the inlet, $C_{\mathrm{g}, \text { in }}^{\mathrm{O}_{2}}$ & 0.21 \\
\hline Net water transport coefficient, $\alpha$ & 0.5 \\
\hline GDL thickness $\left(H_{2}-H_{1}\right)(\mathrm{m})$ & $3.0 \times 10^{-4}$ \\
\hline Channel thickness, $H_{1}(\mathrm{~m})$ & $1.0 \times 10^{-3}$ \\
\hline Liquid water density, $\rho_{1}\left(\mathrm{~m} \mathrm{~kg}^{-3}\right)$ & 974.85 \\
\hline Liquid water kinematic viscosity, $v_{1}\left(\mathrm{~m}^{2} \mathrm{~s}^{-1}\right)$ & $3.65 \times 10^{-7}$ \\
\hline GDL permeability, $K\left(\mathrm{~m}^{2}\right)$ & $5.0 \times 10^{-13}$ \\
\hline Surface tension, $\sigma\left(\mathrm{N} \mathrm{m}^{-1}\right)$ & 0.0625 \\
\hline Cathode transfer coefficient, $\alpha_{\mathrm{c}}$ & 1 \\
\hline Inlet velocity, $u_{\text {in }}\left(\mathrm{m} \mathrm{s}^{-1}\right)$ & 0.4 \\
\hline Channel length, $L(\mathrm{~m})$ & 0.05 \\
\hline
\end{tabular}

with adaptive step size. Some typical parameters used in this work are given in Table 1.

\section{Results and discussion}

The developed two-phase flow model for cathode GDL is used to examine the effects of many important factors on cell performance including the GDL contact angle, porosity, thickness, gas inlet velocity, and channel length. Note that the contact angle $\theta_{\mathrm{c}}$ implies the wettability of the GDL and a larger $\theta_{\mathrm{c}}$ indicates the GDL has a stronger hydrophobicity. Fig. 2 shows the variation of profile of liquid water saturation across the GDL thickness with several assigned values of $\theta_{\mathrm{c}}$ under $I=14,000 \mathrm{~A} \mathrm{~m}^{-2}$. Obviously, the liquid water saturation reduces significantly for a more hydrophobic GDL especially at the interface between the GDL and the catalyst layer (CL). This effect decays gradually with

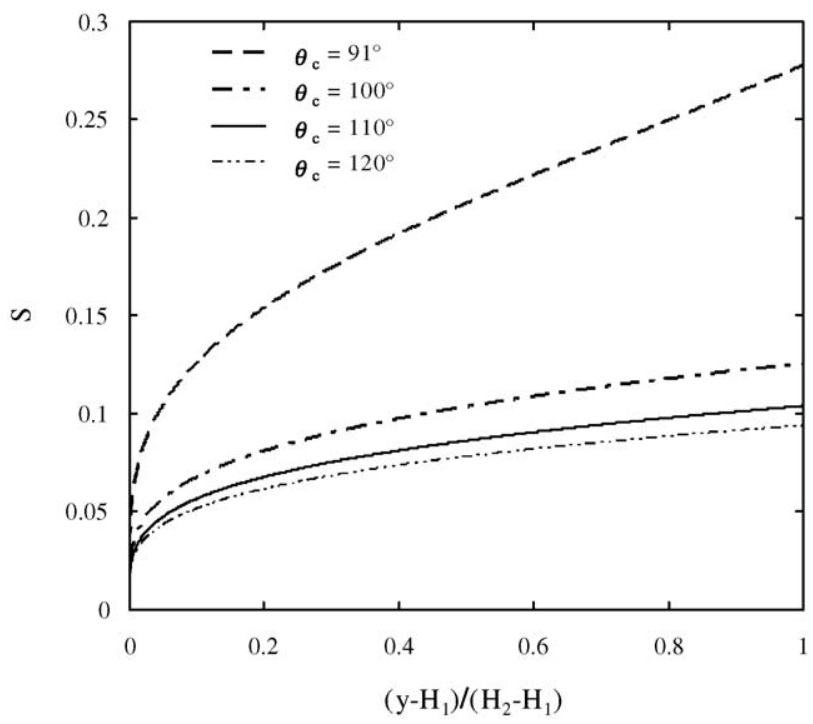

Fig. 2. Profiles of liquid water saturation $s$ across the GDL for several assigned contact angles $\theta_{\mathrm{c}}$ with $I=14,000 \mathrm{~A} \mathrm{~m}^{-2}$.

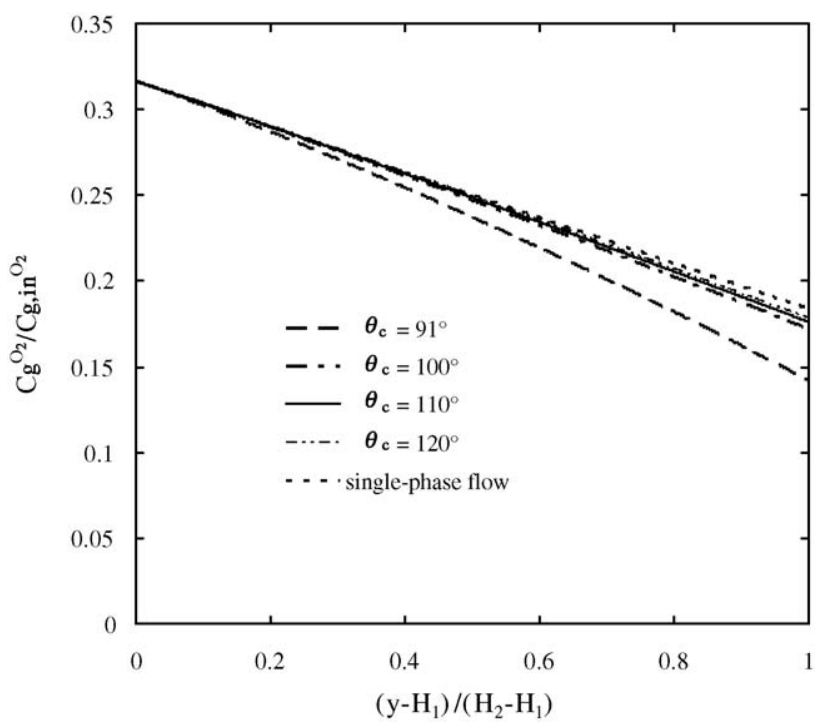

Fig. 3. Profiles of oxygen concentration across the GDL for several different contact angles $\theta_{\mathrm{c}}$ and single-phase flow with $I=14,000 \mathrm{~A} \mathrm{~m}^{-2}$.

increase of $\theta_{\mathrm{c}}$. For example, at the GDL/CL interface, the value of $s$ reduces from 0.278 to 0.126 as $\theta_{\mathrm{c}}$ increases from $91^{\circ}$ to $100^{\circ}$. The percent rate of change of liquid water saturation is about $54.7 \%$. But when $\theta_{\mathrm{c}}$ increases further from $100^{\circ}$ to $110^{\circ}$, the value of $s$ is 0.104 at $\theta_{\mathrm{c}}=110^{\circ}$ and the corresponding percent rate of change of $s$ is about $17.5 \%$ only. Therefore, it should be an efficient way to improve the flooding phenomena by increasing the GDL contact angle, but this effect is limited when $\theta_{\mathrm{c}}$ is large enough. The variation of profile of oxygen concentration is shown in Fig. 3 for several assigned values of $\theta_{\mathrm{c}}$ with $I=14,000 \mathrm{~A} \mathrm{~m}^{-2}$. It is noted that the curve for single-phase flow corresponds to the state where there is no liquid water within the GDL. As seen, oxygen concentration at the GDL/GL interface is lower in the case $\theta_{\mathrm{c}}=91^{\circ}$ because the higher liquid water saturation as shown in Fig. 2 blocks the pores of GDL resulting in an increase of oxygen transport resistance. As $\theta_{\mathrm{c}}$ increases, the removal rate of liquid water rises since the capillary force of GDL becomes greater. Thus, the resistance of oxygen transport reduces and the oxygen concentration at the GDL/CL interface increases with $\theta_{\mathrm{c}}$. Such an effect is apparent at first when $\theta_{\mathrm{c}}$ increases from $91^{\circ}$. As $\theta_{\mathrm{c}}>100^{\circ}$, the profile of oxygen concentration gradually approaches the curve of the single-phase flow and the rise of oxygen concentration by using a GDL with higher $\theta_{\mathrm{c}}$ becomes limited. Fig. 4 illustrates the polarization curves for several assigned values of $\theta_{\mathrm{c}}$. It is found that the influence of liquid water saturation on cell performance is not significant when the cell operates under lower current density conditions. However, at higher current densities, more oxygen is consumed and more water is produced, causing the influence of GDL hydrophobicity to be pronounced. In the case $\theta_{\mathrm{c}}=91^{\circ}$, the higher liquid water saturation makes the mass transport losses occur earlier resulting in a lower limiting current density. Although the limiting current density can be raised by increasing $\theta_{\mathrm{c}}$, it is still less than that in the case of single-phase flow due to the existence of liquid water within the GDL. Furthermore, the increments of the limiting current density between the cases $\theta_{\mathrm{c}}=100^{\circ}, 110^{\circ}$, 


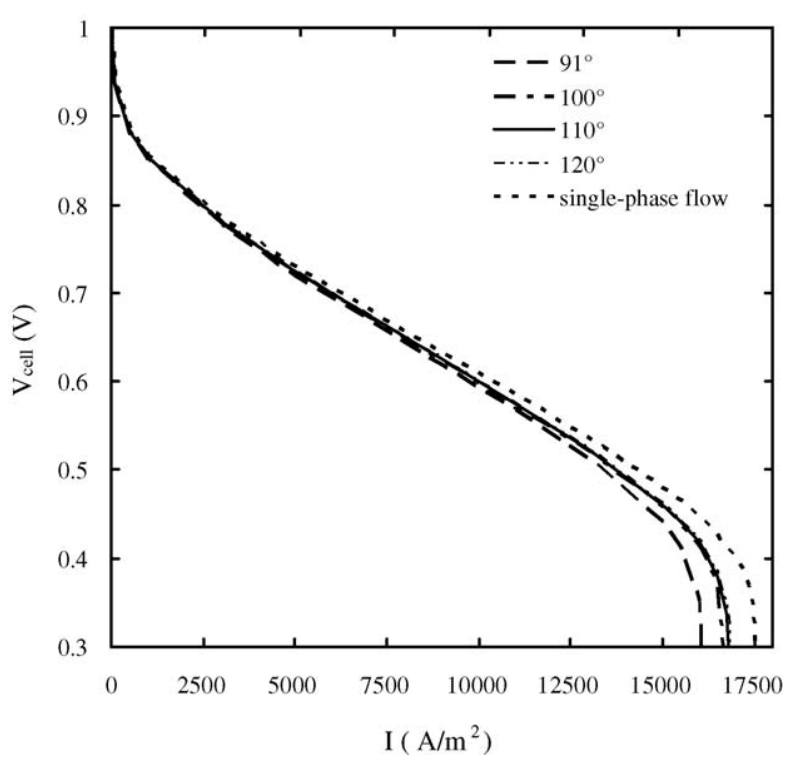

Fig. 4. Polarization curves for several different contact angles $\theta_{\mathrm{c}}$ and singlephase flow.

and $120^{\circ}$ are quite small, which indicates the rate of oxygen transport under such a high current density is almost invariant with $\theta_{\mathrm{c}}$ when $\theta_{\mathrm{c}}$ is sufficiently large.

The effects of GDL porosity on the profile of liquid water saturation are shown in Fig. 5 for three typical values of $\varepsilon$. Both cases $\theta_{\mathrm{c}}=91^{\circ}$ and $110^{\circ}$ are considered with $I=13,000 \mathrm{~A} \mathrm{~m}^{-2}$. For $\theta_{\mathrm{c}}=91^{\circ}$, it is found that the liquid water saturation decreases with an increase of GDL porosity, which indicates that a larger porosity would enhance the removal rate of liquid water in the GDL. But this effect diminishes for a more hydrophobic GDL as shown in the case $\theta_{\mathrm{c}}=110^{\circ}$. Therefore, the porosity may influence the liquid water saturation profile significantly only when the GDL has a lower hydrophobicity.

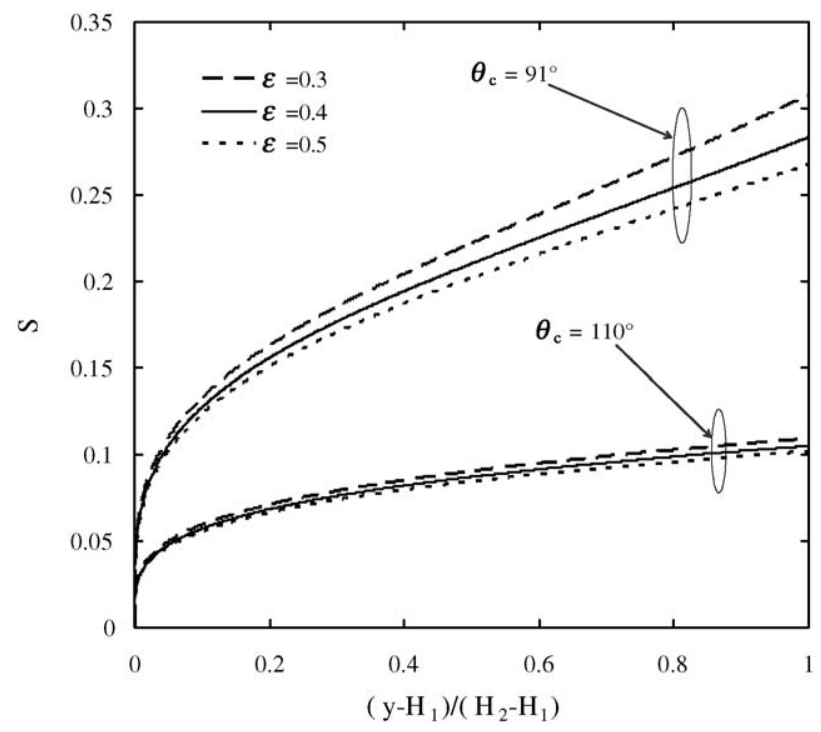

Fig. 5. Profiles of liquid water saturation $s$ across the GDL for both cases of $\theta_{\mathrm{c}}$ with three assigned GDL porosities under $I=13,000 \mathrm{~A} \mathrm{~m}^{-2}$.

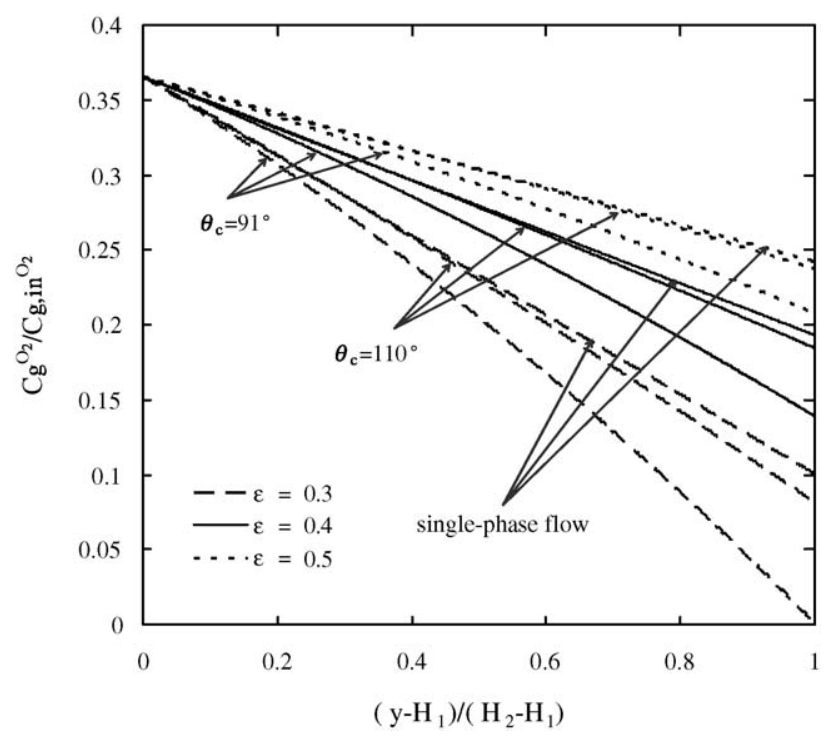

Fig. 6. Profiles of oxygen concentration across the GDL for both cases of $\theta_{\mathrm{c}}$ and single-phase flow with three assigned GDL porosities under $I=13,000 \mathrm{~A} \mathrm{~m}^{-2}$.

In contrast to the small influence on liquid water saturation profile, the GDL porosity plays an important role in the variation of oxygen concentration profile as demonstrated in Fig. 6. A larger porosity significantly reduces the resistance of oxygen transport through the GDL and greatly raises the oxygen concentration on the GDL/CL interface due to the increase of effective diffusion coefficient as indicated in Eq. (33). For example, the oxygen concentration adjacent to the catalyst layer in the case $\varepsilon=0.3$ with $\theta_{c}=91^{\circ}$ is almost consumed thoroughly by the electrochemical reaction. The insufficient supply of oxygen causes severe concentration losses and restricts the cell performance. Such phenomena can be improved if the GDL material is replaced by a higher porosity one as shown in the cases $\varepsilon=0.4$ and 0.5 . Similar results also can be observed in the cases $\theta_{\mathrm{c}}=110^{\circ}$ and the single-phase flow model. It also can be predicted from these results that the limiting current density can be enhanced by a larger porosity for a given GDL contact angle $\theta_{\mathrm{c}}$. Fig. 7 shows the corresponding polarization curves for these three typical porosities $\varepsilon=0.3,0.4$, and 0.5 , and each case contains three typical cases $\theta_{\mathrm{c}}=91^{\circ}, 110^{\circ}$, and the model of single-phase flow. Apparently, the effects of two-phase flow are important when the fuel cell operates under high current density conditions and the cell performance is better with higher porosity.

The thickness of cathode GDL is also an important factor to affect the cell performance. Fig. 8 illustrates the profiles of liquid water saturation for three different thicknesses 200,300, and $400 \mu \mathrm{m}$. Both typical contact angles $\theta_{\mathrm{c}}=91^{\circ}$ and $110^{\circ}$ are considered with current density $I=14,000 \mathrm{~A} \mathrm{~m}^{-2}$. The results show that if the GDL is less hydrophobic (i.e. $\theta_{\mathrm{c}}=91^{\circ}$ ), the lower removal rate of liquid water causes a high liquid water saturation on the GDL/CL interface. Moreover, a thicker GDL leads to a higher resistance for liquid water transport. As a result, the liquid water saturation rises rapidly with increasing GDL thickness. However, if the GDL is more hydrophobic (i.e. $\theta_{\mathrm{c}}=110^{\circ}$ ), the capillarity-induced flow dominates the liquid water transport 

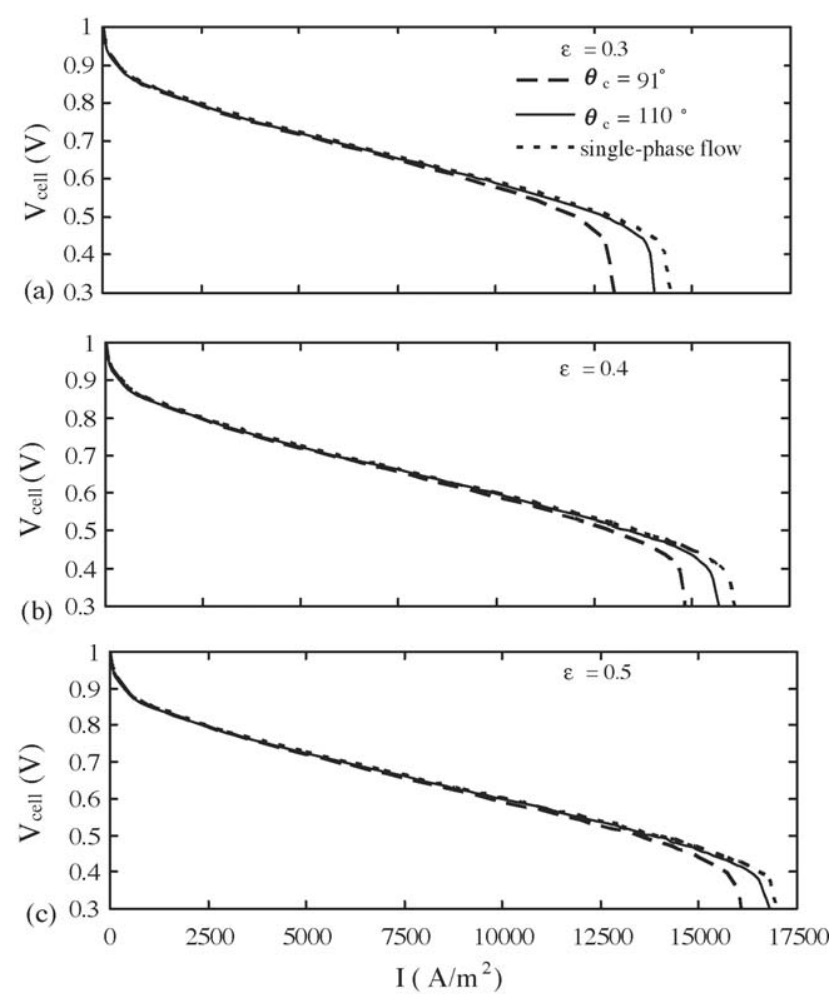

Fig. 7. Variation of polarization curves with contact angle $\theta_{\mathrm{c}}$ for three assigned GDL porosities: (a) $\varepsilon=0.3$, (b) $\varepsilon=0.4$, and (c) $\varepsilon=0.5$.

and the removal rate is increased. Therefore, the liquid water saturation on the GDL/CL interface reduces and the variation of GDL thickness only produces a slight influence on the profile of liquid water saturation.

The effects of GDL thickness on the profile of oxygen concentration are demonstrated in Fig. 9 for the same cases considered in Fig. 8 and the case of single-phase flow. As shown in this figure, the oxygen transport is profoundly affected by the GDL

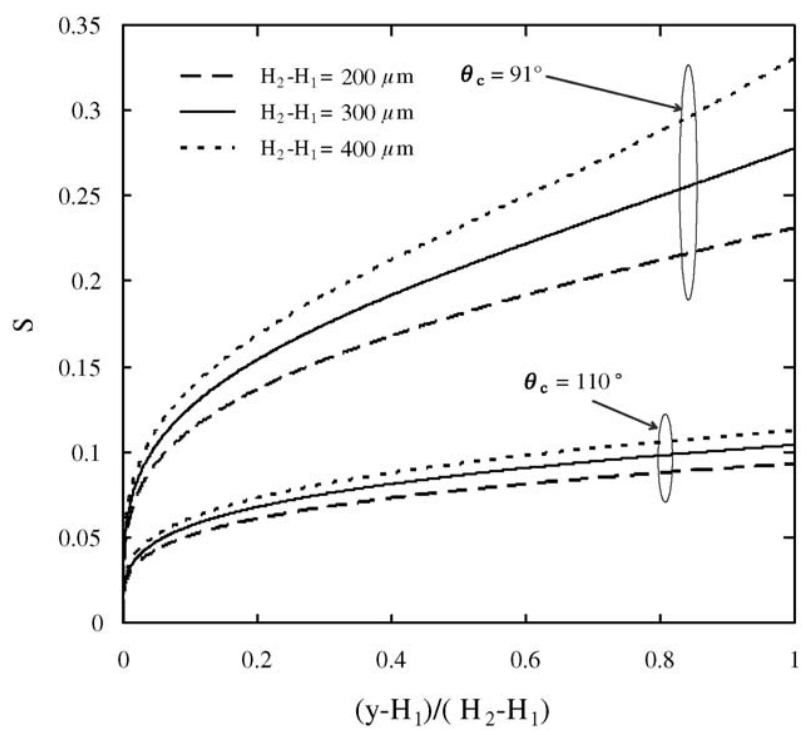

Fig. 8. Profiles of liquid water saturation $s$ across the GDL for both cases of $\theta_{\mathrm{c}}$ with three assigned GDL thicknesses under $I=14,000 \mathrm{~A} \mathrm{~m}^{-2}$.

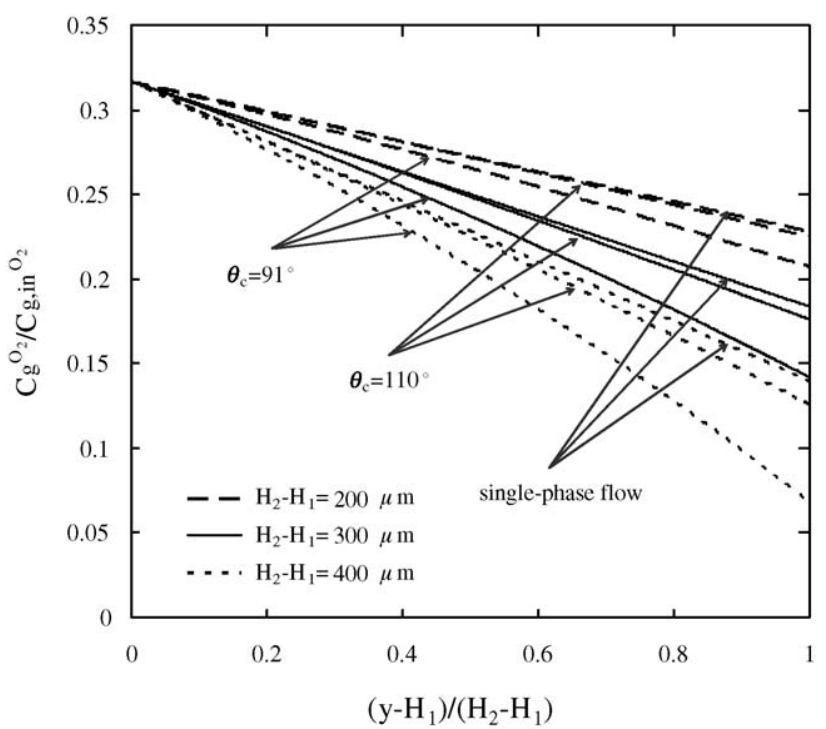

Fig. 9. Profiles of oxygen concentration across the GDL for both cases of $\theta_{\mathrm{c}}$ and single-phase flow with three assigned GDL thicknesses under $I=14,000 \mathrm{~A} \mathrm{~m}^{-2}$.

thickness. In the case $\theta_{\mathrm{c}}=91^{\circ}$, the oxygen concentration near the catalyst layer decreases rapidly with an increase of GDL thickness. Consequently, the concentration losses due to insufficient mass transport of oxygen take place in advance for a thicker GDL. Similar results also can be observed in the cases $\theta_{\mathrm{c}}=110^{\circ}$ and the single-phase flow model. An oxygen concentration difference is noticed on the GDL/CL interface between the case $\theta_{\mathrm{c}}=91^{\circ}$ and the single-phase flow model, and this difference gradually grows with GDL thickness. This result indicates that the influence of two-phase flow is less important for a thinner GDL, while it becomes a critical factor for a thicker GDL in fuel cell performance. The corresponding polarization curves for these three typical GDL thicknesses are shown in Fig. 10. Apparently, the cell performance is lower for a thicker GDL since the limiting current density decreases with GDL thickness.

The influences of gas inlet velocity and gas channel length also can be examined via the developed theoretical model. Fig. 11 demonstrates the variation of polarization curves for three assigned inlet velocities. In the case $u_{\text {in }}=0.2 \mathrm{~m} \mathrm{~s}^{-1}$, the volumetric gas flow rate is too low to provide sufficient oxygen through the GDL for electrochemical reaction. Therefore, the concentration losses occur early and make the lower limiting current density. In addition, the generation of liquid water is too slow to produce a significant effect on blocking the GDL. Hence, the liquid phase plays a less important role when the gas inlet velocity is small, which can be interpreted by the little difference between the limiting current densities of the curves for the case $\theta_{\mathrm{c}}=91^{\circ}$ and the single-phase flow model. As $u_{\text {in }}$ increases, more oxygen can be transported to the catalyst layer for electrochemical reaction. As a result, the cell performance enhances and the limiting current density rises obviously as shown in the case $u_{\text {in }}=0.4 \mathrm{~m} \mathrm{~s}^{-1}$. Furthermore, the influence of liquid water grows gradually when the cell operates under high current densities especially for a less hydrophobic GDL. One can observe that the difference of limiting current density between the case 

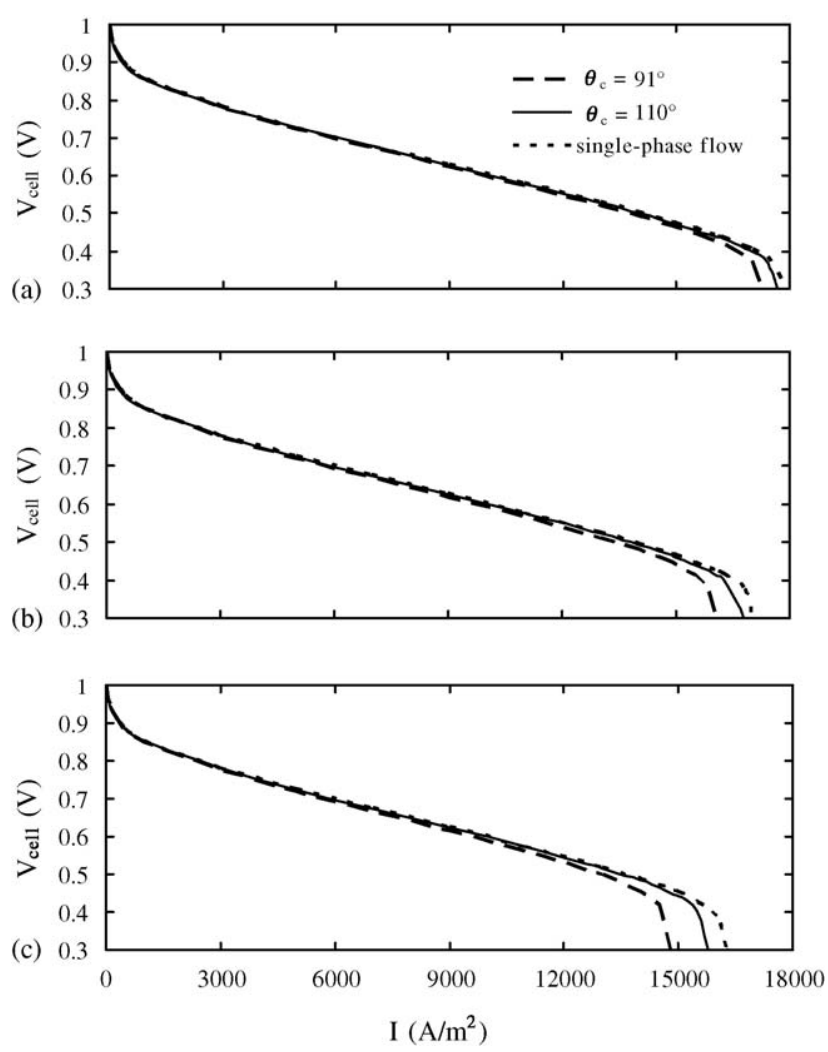

Fig. 10. Variation of polarization curves with contact angle $\theta_{\mathrm{c}}$ for three assigned GDL thicknesses: (a) $H_{2}-H_{1}=200 \mu \mathrm{m}$, (b) $H_{2}-H_{1}=300 \mu \mathrm{m}$, and (c) $H_{2}-H_{1}=400 \mu \mathrm{m}$.

$\theta_{\mathrm{c}}=91^{\circ}$ and the single-phase model becomes distinct, and such a difference enlarges further in the case $u_{\mathrm{in}}=0.6 \mathrm{~m} \mathrm{~s}^{-1}$.

Fig. 12 shows the variation of polarization curves for three different gas channel lengths. For the shorter channel length case with $L=5 \mathrm{~cm}$, the fuel cell exhibits a better performance due to the higher average oxygen concentration along the channel as well as the electrochemical reaction rate. The higher limiting

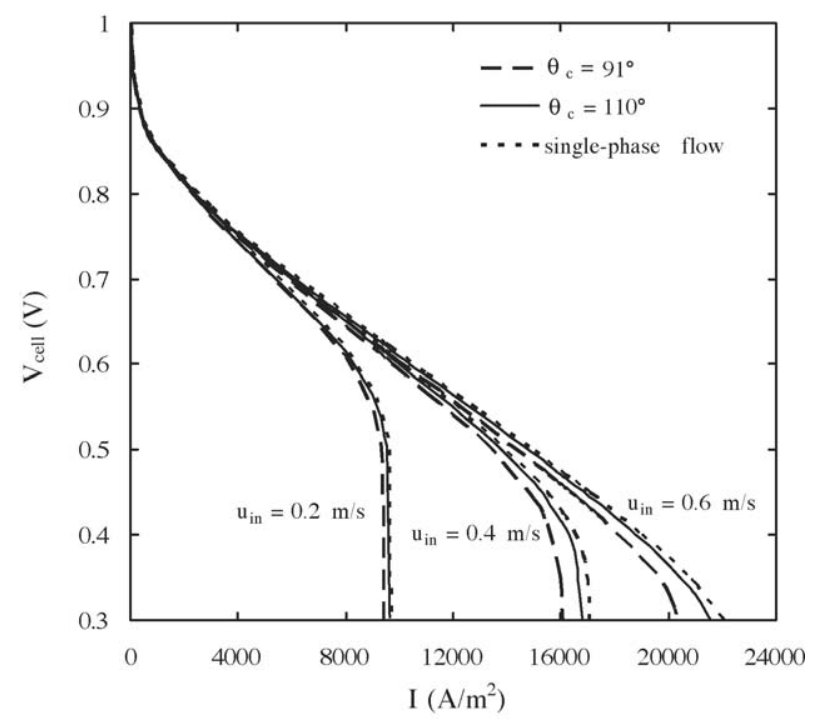

Fig. 11. Variation of polarization curves for three assigned inlet velocities with both contact angles $\theta_{\mathrm{c}}$ and single-phase flow.

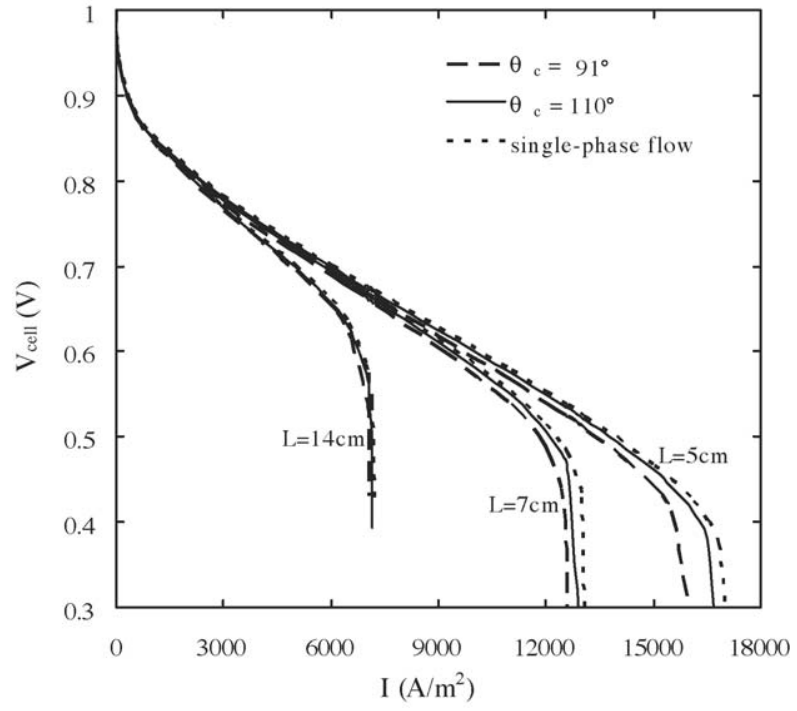

Fig. 12. Variation of polarization curves for three assigned gas channel lengths with both contact angles $\theta_{\mathrm{c}}$ and single-phase flow.

current density simultaneously accompanies a faster water generation rate resulting in higher liquid water saturation in the GDL. Accordingly, the effective porosity of the GDL reduces as well as the oxygen transport, so the limiting current densities of both cases $\theta_{c}=91^{\circ}$ and $110^{\circ}$ are distinctly less than that of the single-phase flow model. As the channel length increases, the mean oxygen concentration along the channel descends quickly which causes a great reduction of limiting current density as shown in the cases $L=7$ and $14 \mathrm{~cm}$. The effect of liquid water saturation also decreases gradually. For $L=14 \mathrm{~cm}$, the three curves almost overlap, which indicates the liquid water saturation is quite small and the results are equivalent to those of the singlephase flow model.

\section{Conclusions}

A two-phase flow analysis has been implemented to examine the characteristics of transport phenomena in the cathode GDL of a PEMFC and their influences on cell performance. The developed theoretical model provides a simple way to estimate the effects of the gas inlet velocity and gas channel length. It was known that a hydrophobic GDL possesses a higher removal rate for liquid water to prevent the flooding phenomenon. Therefore, this study considered the hydrophobic GDL with $\theta_{\mathrm{c}}>90^{\circ}$ only and found that the liquid water saturation indeed is reduced significantly under high current density conditions when $\theta_{\mathrm{c}}$ increase from $91^{\circ}$ and the oxygen transport is enhanced due to the improvement of effective porosity of the GDL. However, this effect decays gradually with $\theta_{\mathrm{c}}$ and is limited if $\theta_{\mathrm{c}}$ is large enough. The profiles of liquid water saturation and oxygen concentration are sensitive to the porosity and thickness of GDL. It is found that a smaller porosity and a thicker GDL produce the same effect to raise the resistance of oxygen transport and lower the cell performance.

The gas inlet velocity and the channel length are both important factors that affect the oxygen concentration along the chan- 
nel as well as in the GDL. The results show that the concentration losses are serious for a smaller inlet velocity or a longer channel length. The generation of liquid water is not significant in these cases due to the lower limiting current densities. However, an increase of inlet velocity or a shorter channel length can enhance the cell performance obviously, and the two-phase flow in GDL exhibits an important influence on the variation of limiting current density. These results are beneficial to the design of a PEMFC with regard to water management problems.

\section{Acknowledgments}

The financial support for this research from National Science Council of Taiwan through the grants NSC 94-2212-E-132005, NSC 94-2212-E-002-009, and NSC 94-2622-E-002-004 are gratefully acknowledged.

\section{References}

[1] D.M. Bernardi, J. Electrochem. Soc. 137 (1990) 3344-3350.

[2] D.M. Bernardi, M.W. Verbrugge, AIChE J. 37 (1991) 1151-1163.

[3] T.E. Springer, T.A. Zawodzinski, S. Gottesfeld, J. Electrochem. Soc. 138 (1991) 2334-2342.
[4] D.M. Bernardi, M.W. Verbrugge, J. Electrochem. Soc. 139 (1992) 2477-2490.

[5] T.F. Fuller, J. Newman, J. Electrochem. Soc. 140 (1993) 1218-1225.

[6] D. Singh, D.M. Lu, N. Djilali, Int. J. Eng. Sci. 37 (1999) 431-452.

[7] J.J. Baschuk, X. Li, J. Power Sources 86 (2000) 181-196.

[8] A. Rowe, X. Li, J. Power Sources 102 (2001) 82-96.

[9] T. Berning, D.M. Lu, N. Djilali, J. Power Sources 106 (2002) 284-294.

[10] J.H. Nam, M. Kaviany, Int. J. Heat Mass Transfer 46 (2003) 4595-4611.

[11] N.P. Siegel, M.W. Ellis, D.J. Nelson, M.R. von Spakovsky, J. Power Sources 128 (2004) 173-184.

[12] W. He, J.S. Yi, T.V. Nguyen, AIChE J. 46 (2000) 2053-2064.

[13] T. Berning, N. Djilali, J. Electrochem. Soc. 150 (2003) 1589-1598.

[14] C.Y. Wang, P. Cheng, Int. J. Heat Mass Transfer 39 (1996) 3607-3618.

[15] C.Y. Wang, P. Cheng, Adv. Heat Transfer 30 (1997) 93-196.

[16] Z.H. Wang, C.Y. Wang, K.S. Chen, J. Power Sources 94 (2001) 40 50.

[17] D. Natarajan, T.V. Nguyen, J. Electrochem. Soc. 148 (2001) 1324-1335.

[18] L. You, H. Liu, Int. J. Heat Mass Transfer 45 (2002) 2277-2287.

[19] U. Pasaogullari, C.Y. Wang, J. Electrochem. Soc. 151 (2004) 399-406.

[20] U. Pasaogullari, C.Y. Wang, Electrochim. Acta 49 (2004) 4359-4369.

[21] U. Pasaogullari, C.Y. Wang, K.S. Chen, J. Electrochem. Soc. 152 (2005) A1574-A1582.

[22] M. Kaviany, Principle of Heat Transfer in Porous Media, Springer, New York, 1995.

[23] R.E. Meredith, C.W. Tobias, Advances in Electrochemical Science and Engineering, 2nd ed., Interscience, New York, 1962. 